\title{
Highly Reliable and Low-Cost Fabrication of Warm-White LEDs Using Composite Silica Photonic Crystals
}

\author{
Chun-Feng Lai, ${ }^{1}$ Yu-Chun Lee, ${ }^{2}$ Tzong-Liang Tsai, ${ }^{2}$ \\ Chung-Chieh Chang, ${ }^{3}$ and Mau-Kuen $\mathrm{Wu}^{3}$ \\ ${ }^{1}$ Department of Photonics, Feng Chia University, 100 Wenhwa Road, Seatwen, Taichung 40724, Taiwan \\ ${ }^{2}$ Lextar Electronics Corp., No. 3, Gongye E. 3rd Road, Hsinchu Science Park, Hsinchu 30075, Taiwan \\ ${ }^{3}$ Institute of Physics, Academia Sinica, Nankang, Taipei 11529, Taiwan \\ Correspondence should be addressed to Chun-Feng Lai; chunflai@fcu.edu.tw
}

Received 11 July 2014; Accepted 14 November 2014; Published 3 December 2014

Academic Editor: Mark van Der Auweraer

Copyright (C) 2014 Chun-Feng Lai et al. This is an open access article distributed under the Creative Commons Attribution License, which permits unrestricted use, distribution, and reproduction in any medium, provided the original work is properly cited.

\begin{abstract}
We demonstrated a technique requiring little phosphor that used white light-emitting diodes (WLEDs) containing composite silica colloidal photonic crystals ( $c$-SCPhCs) for developing the warm-WLEDs ( $w$-WLEDs). WLEDs containing $c$-SCPhCs enhanced luminous efficacy 5.6\% more than commercial $w$-WLEDs did. We used a UV adhesive curing method to improve the adhesion properties of the $c$-SCPhCs. A reliability analysis (RA) test was performed on the WLEDs containing $c$-SCPhCs, applying a high temperature and high relative humidity $\left(85^{\circ} \mathrm{C} / 85 \mathrm{RH}\right)$ during WLEDs operation at $120 \mathrm{~mA}$. During a RA test of $2500 \mathrm{~h}$, no visible degradation in optical performance was observed. We implemented a novel, inexpensive technique for producing high luminous flux $w$-WLEDs that can be used in residential light.
\end{abstract}

\section{Introduction}

Recently, solid-state lighting devices, such as white lightemitting diodes (WLEDs) and organic LEDs (OLEDs), have been rapidly developed [1-4] as the predominant lighting used for new lighting installations. Because of their multiple advantages, such as high brightness, high reliability, low power consumption, versatility, and robustness, WLEDs have produced a wide range of benefits and opportunities for the lighting industry. The correlated color temperature (CCT) of commercial warm-WLEDs ( $w$-WLEDs), which are a particularly valuable light source because they provide comfortable indoor lighting, is approximately 2700 to $3000 \mathrm{~K}$. Commercially available WLEDs generally use a GaN-based blue LED for stimulating yellow phosphor (YAG:Ce) to yield phosphor-converted WLEDs (PC-WLEDs), which are the most efficient lights that provide high luminous efficiency at a low cost [5]. Although PC-WLEDs have numerous advantages, such as ease of fabrication, low cost, and a phosphor conversion efficiency that exceeds $70 \%$, several PC-WLEDs problems remain, including a high CCT, a low color-rendering index (CRI), and the absence of a red spectrum [6]. Therefore, phosphors of two or more colors are required when the high CCT of WLEDs decrease to the low CCT of $w$-WLEDs. Currently, all nitride- and boratebased red-emitting phosphors are typically 5 to 10 times more expensive per gram than yellow-emitting phosphors, a phenomenon that substantially contributes to the high cost of manufacturing $w$-WLEDs. Reducing the cost of $w$-WLEDs can hasten the transition from conventional bulbs to LEDs and lead to new applications in optical devices.

Previously, the light emission spectrum of WLEDs has been modified using 3D polystyrene (PS) colloidal photonic crystals (CPhCs) to resemble $w$-WLEDs and candlelight [79]; however, the PS material has a glass transition temperature $\left(T_{g}\right)$ of approximately $90^{\circ} \mathrm{C}$, causing the WLEDs with composite PS CPhC to be unreliable in high temperature environments. To resolve the aforementioned problems, in this study, we deposited WLEDs with composite silica $\mathrm{CPhC}$ (c-SCPhC) thin films to fabricate low-cost $w$-WLEDs. A silica nanosphere was used to solve the $T_{g}$ and a UV adhesive curing method was applied to enhance the adhesion 
properties of the SCPhCs. The light emission spectrum of WLEDs containing $c$-SCPhCs was modified to resemble $w$-WLEDs by using the photonic band structure (PBS) of $3 \mathrm{D} c$-SCPhCs. Thus, the WLED CCT of approximately $4000 \mathrm{~K}$ decreased to the $w$-WLED CCT of approximately $3000 \mathrm{~K}$ without increasing the weight percentage (wt\%) of the phosphor layer, and the luminous efficacy was enhanced. In addition, a method to test the reliability of the WLEDs containing $c$-SCPhCs was applied in this study.

\section{Experiments}

Commercial WLEDs and $w$-WLEDs that produced a CCT of approximately $4000 \mathrm{~K}$ and $3000 \mathrm{~K}$, respectively, were fabricated by Lextar Electronics Corp. in Taiwan. In the current study, the WLEDs and $w$-WLEDs were fabricated as follows. First, a $35 \times 22 \mathrm{mil}^{2}$ chip of GaN-based patterned sapphire substrate (PSS) LEDs (emission wavelength, $445 \mathrm{~nm}$ ) was bonded to a commercial 5630-type plastic-leaded chip carrier (PLCC) and packaged using silver paste and gold wire. The GaN PSS LED structures used in this study were identical to those described previously [7]. Subsequently, a green phosphor $\left(\mathrm{Lu}_{3} \mathrm{Al}_{5} \mathrm{O}_{12}: \mathrm{Ce}^{3+}\right.$, LuAG) and a red phosphor $\left(\mathrm{CaAlSiN}_{3}: \mathrm{Eu}^{2+}, \mathrm{CASN}\right)$ with various wt $\%$ concentrations were used to create the WLEDs and $w$-WLEDs. The WLEDs had a LuAG and CASN phosphor concentration of $21.4 \mathrm{wt} \%$ and $3.6 \mathrm{wt} \%$, respectively, and the $w$-WLEDs had a LuAG and CASN phosphor concentration of $23.1 \mathrm{wt} \%$ and $4.5 \mathrm{wt} \%$, respectively. They were uniformly mixed with silicone and then poured into the lead frame by using traditional phosphor-dispensing techniques. For the WLEDs to become $w$-WLEDs, the LuAG phosphor concentration must be increased by $8 \%$ and the CASN phosphor must be increased by $25 \%$, an increase which raises the fabrication cost of $w$-WLEDs.

Next, we prepared the latex $(100.0 \mathrm{mg} / \mathrm{mL})$ by using a silica nanosphere with a diameter $(D)$ of $400 \mathrm{~nm}$. The SCPhCs $(3.0 \mu \mathrm{L})$ were deposited onto the entire emission region of the WLEDs by employing a micropipette and a vertical deposition method [10]. Monodispersed silica nanospheres $(D=400 \mathrm{~nm})$ were synthesized using ammonium hydroxide $\left(\mathrm{NH}_{4} \mathrm{OH}, 5.0 \mathrm{~mL}\right)$ and deionized (DI) water $(20.0 \mathrm{~mL})$, and the tetraethoxysilane (TEOS, $5.0 \mathrm{~mL}$; Aldrich, 98\%) condensation was controlled in an ethanol (EtOH, $50.0 \mathrm{~mL})$ solution $[11,12]$. Furthermore, the interstitial structure of the SCPhCs caused the colloidal nanospheres to adhere ineffectively. Therefore, to enable the SCPhCs to be reliable in test conditions, a photocurable resin of a UV adhesive solution was used as a dispersion medium, enhancing the linkage among the colloidal silica nanospheres [13-15]. A simple method was developed using the UV adhesive solution to prepare $c$ SCPhCs that exhibited high optical transparency and physical rigidity. The crosslinked UV adhesive enhanced the interactions among the silica nanospheres in the SCPhC thin film, thereby improving the adhesion. We used an optimal volume of $1.0 \mu \mathrm{L}$ for the UV adhesive solution, which infiltrated the interstitial spaces in the SCPhC thin films [9]; a solution with a concentration of $9.1 \mathrm{wt} \%$ was prepared by mixing
$1.0 \mathrm{mg}$ of commercial UV adhesive and $10.0 \mathrm{~mL}$ of an acetone solution. After the UV adhesive solution had infiltrated the $\mathrm{SCPhC}$ thin film and entered the gaps under the capillary forces, we exposed the sample to UV light for $10 \mathrm{~min}$ to ensure that the photopolymerization of the UV adhesive was complete. Figures 1(a) and 1(b) show the optical microscopy image and schematic diagram, respectively, of the $c$-SCPhCs deposited onto the 5630-type PLCC-packaged WLED. The optical microscopy image in Figure 1(a) was taken in natural lighting conditions, revealing the iridescent-dependent Bragg reflection. We used the field-emission scanning electron microscopy (FESEM) to investigate the crystalline structure of the $c$-SCPhCs on the WLEDs. Figures 1(c) and 1(d) show images of the outer (111) and inner (100) faces, respectively, of the $c$-SCPhCs with face-centered cubic structures [16]. Figure 1(c) illustrates that the UV adhesive solution infiltrated the interstices of the silica nanospheres. This simple approach improved the adhesion of the $c$-SCPhCs, thereby enhancing their practical application.

The $c$-SCPhCs feature the PBS properties of the 3D PhCs $[11,12]$. The Bragg reflection wavelength $\left(\lambda_{R}\right)$ of $c$-SCPhCs can be calculated according to the Bragg law [17]:

$$
\lambda_{R}=2 d_{111} \sqrt{n_{\mathrm{eff}}^{2}-\sin ^{2} \theta_{111}}
$$

where $\lambda_{R}$ is the wavelength of the reflection peak, $d_{111}$ is the interplanar spacing between the (111) planes, and $n_{\text {eff }}$ is the effective refractive index of the crystalline lattice. Regarding the $c$-SCPhC structure, $n_{\text {eff }}$ was obtained using

$$
n_{\mathrm{eff}}^{2}=n_{\text {Silica }}^{2} f_{\text {Silica }}+n_{\mathrm{UV}}^{2}\left[1-f_{\text {Silica }}\right]
$$

where $n_{\text {Silica }}=1.5$ and $n_{\mathrm{UV}}=1.1$ are the refractive indices of the silica nanospheres and UV adhesive solution, respectively; $f_{\text {Silica }}=0.74$ is the volume fraction of the nanosphere [9]. The normal reflection spectrum for this $c$ SCPhC exhibited a reflection peak at $923 \mathrm{~nm}$ that adhered to the Bragg law (Figure 2(a)). The Bragg reflection light shifted toward a shorter wavelength when the incident angle increased [7-9]. Therefore, the angular-resolved reflection spectra were measured to examine the light-reflection distribution of the $c$-SCPhCs; Figure 2(b) shows the PBS of the $3 \mathrm{D} c$-SCPhCs. The angular-resolved reflection measurement system used in this study was identical to that described previously [9]. To confirm that the high-order band of PBS affected the luminescence spectrum of the WLED, we measured the transmittance spectrum of the $c-S C P h C$ thin films by using a UV-Vis spectrophotometer (Figure 2(c)). We used a transmittance measurement system equipped with a halogen lamp as the white light source, an integration sphere, and a spectrometer with a charge-coupled device to measure the transmittance spectra through the $c$-SCPhC thin films. The $c$-SCPhC thin films exhibited a graduated transmission from the blue wavelength region to the red wavelength region. The transmittance of $c$-SCPhC thin films was similar to that of the graduated neutral density filter (GNDF). The 400-600 nm wavelength spanning from the blue to the red region exhibited a low transmittance that was affected by the high-order band of PBS (Figure 2(b)). 


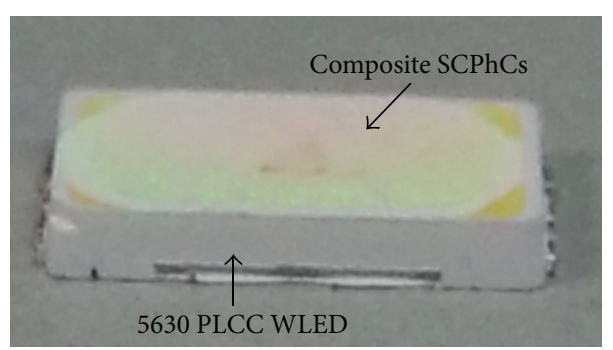

(a)

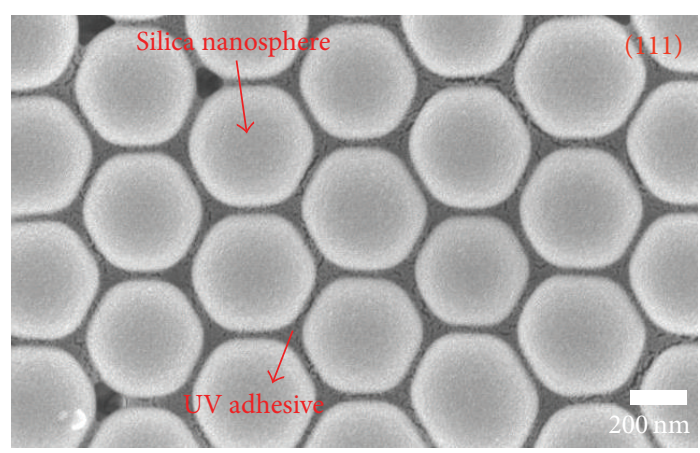

(c)

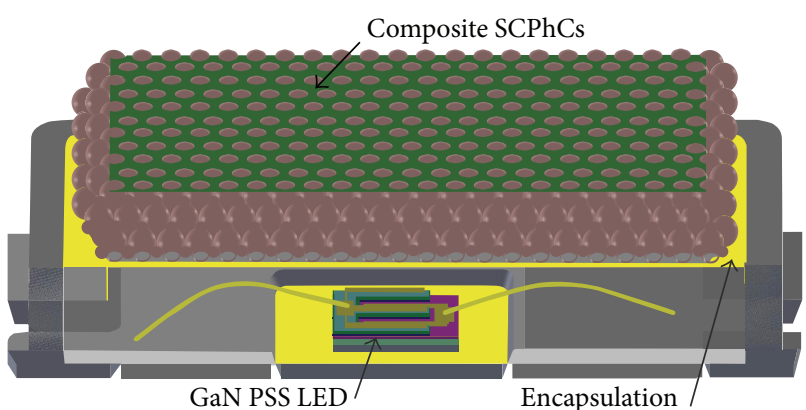

(b)

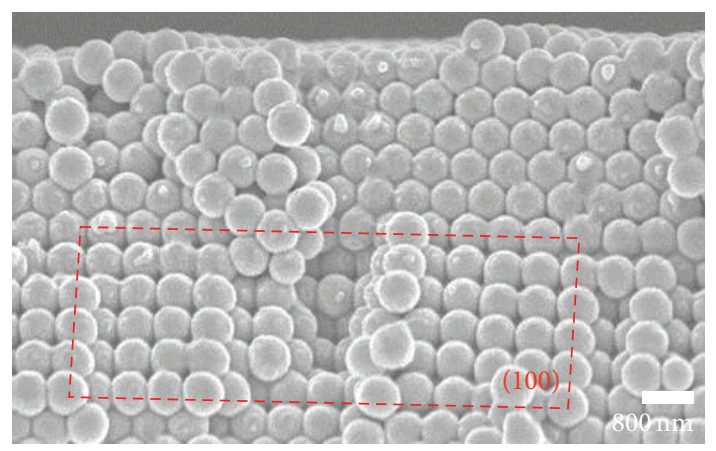

(d)

FIGURE 1: (a) Optical microscopy image and (b) schematic diagram of the $c$-SCPhCs deposited onto the 5630-type PLCC-packaged WLEDs. FESEM images of the $c$-SCPhCs prepared using silica nanospheres with a diameter of $400 \mathrm{~nm}$; (c) and (d) display a well-organized (111) plane and (100) plane of face-centered cubic structures.

In other words, when the WLED photons passed through the $c$-SCPhC thin films, one part was reflected and the other was transmitted. The reflected light of the WLEDs can be absorbed by the phosphor layer and increase the red-light emission [7-9]. In this paper, we report that the WLED devices containing $c$-SCPhCs achieved a higher luminous flux than the commercial $w$-WLEDs, because of the GNDF influence and increased light reemission in the red-light region (Figure 3).

\section{Results and Discussion}

The $c$-SCPhC thin films modified the luminescence spectra of the WLEDs, which were measured using a 20 in integration sphere with a current setting of $120 \mathrm{~mA}$, as shown in Figure 3; the $425-600 \mathrm{~nm}$ light emission wavelengths of the WLEDs containing $c$-SCPhCs were suppressed by the $c$-SCPhC thin films. In addition, the red-light wavelength exceeding $600 \mathrm{~nm}$ of the WLEDs containing $c$-SCPhCs increased (Figure 3) because of the reabsorption and reemission produced by the phosphor layer of the WLEDs [7-9]. The reflected light was incidental to the package surfaces, indicating a conversion of reflected light into the phosphor layer of the WLEDs. $w$-WLEDs constantly require adequate red light; therefore, recycling as much red light as possible before it is absorbed is essential to improving the luminous efficacy.
In this experiment, we measured the luminous flux, luminous efficacy, CRI, and CCT in relation to current characteristics by using the 20 in integration sphere (purchased from Labsphere) that was equipped with a radiometer and photometer (SC-610). Figure 4 and Table 1 show that, at an input power of $0.37 \mathrm{~W}$, the luminous flux values of the WLEDs, $w$-WLEDs, and WLEDs containing $c$-SCPhCs were 53.2, 44.3, and $46.7 \mathrm{~lm}$, respectively. The luminous efficacy of these WLEDs was 144,120 , and $127 \mathrm{~lm} / \mathrm{W}$, respectively. The luminous flux of the WLEDs containing $c$-SCPhCs was $5.6 \%$ more enhanced than that of the $w$-WLEDs. To confirm this enhanced luminous flux, we measured the luminous flux of 10 random samples obtained from commercial WLEDs and $w$-WLEDs that were fabricated by Lextar Electronics Corp. According to the results, the true percent relative error $\left(\varepsilon_{t}\right)$ of the WLEDs and $w$-WLEDs was approximately $1.22 \%$ and $1.46 \%$, respectively. The maximal $\varepsilon_{t}$ of these samplings did not exceed $4 \%$. Therefore, the luminous flux of the WLEDs containing $c$-SCPhCs was higher than that of the $w$-WLEDs. Additionally, the CCT of WLEDs containing $c$ SCPhCs decreased from approximately $3974 \mathrm{~K}$ to $2960 \mathrm{~K}$ without an increase in the wt $\%$ of the LuAG and CASN phosphors. Table 1 lists the optical properties of all light sources and the International Commission on Illumination (CIE) color chromaticity coordinates $(x, y)$, which delineate numerous standard illuminants used as colorimetric references. The CIE $(x, y)$ coordinates of all light sources in this study were located near the Planckian locus of the 


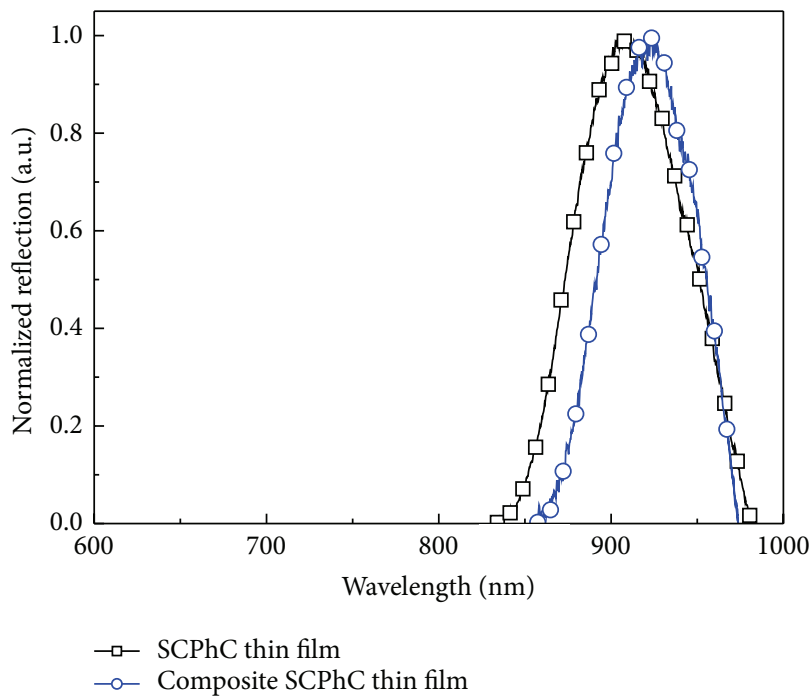

(a)

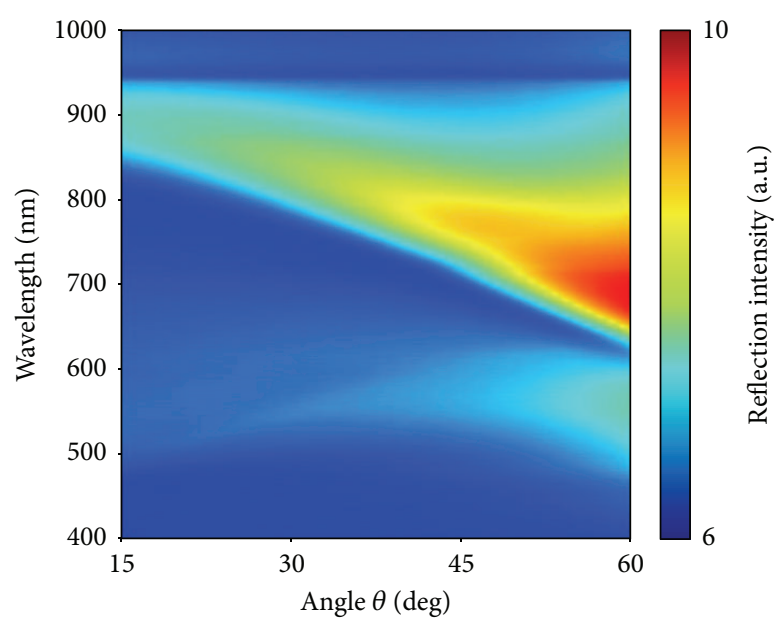

(b)

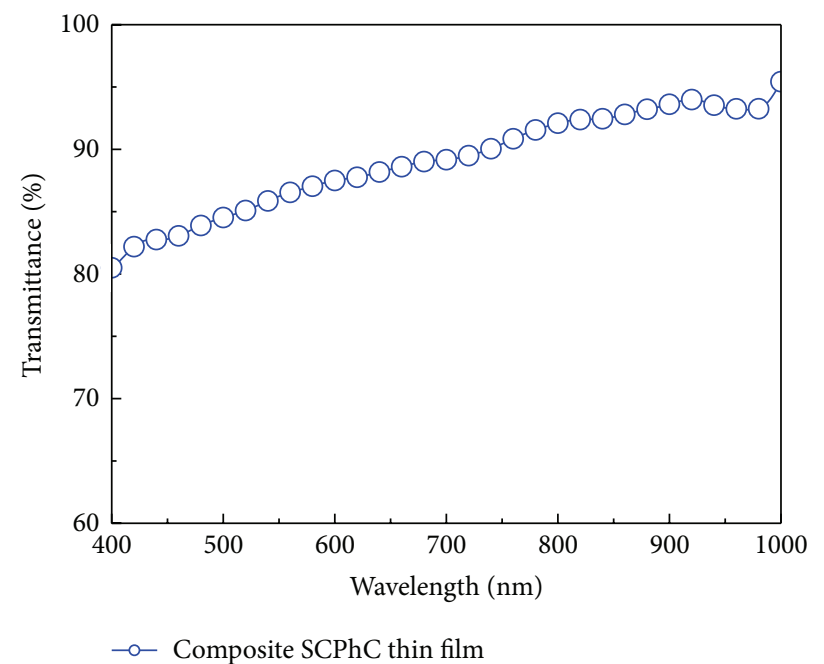

(c)

FIGURE 2: (a) Peak positions of the reflection spectra measured from the SCPhCs were 910 and 923 nm, respectively, before and after photocrosslinking was performed using a UV adhesive solution. (b) Unpolarized angular-resolved reflection measurements of $c$-SCPhC thin films. (c) Measured transmittance of the $c$-SCPhC thin films.

CIE chromaticity diagram. All WLEDs must have a CRI that exceeds 80, a standard that is required for use in general illumination applications. In this study, a novel $w$-WLED fabrication technique that reduces the cost of fabricating $w$-WLEDs by approximately $17 \%$, which was calculated by Lextar Electronics Corp., was developed.

The reliability analysis (RA) test [18], which was experimented with by Lextar Electronics Corp., is crucial for the application of WLED devices. The RA test is generally used to determine the operation time during which the output luminous flux of WLED devices decreases to $70 \%$ of the initial value of the WLED devices. We used a wet, high temperature operating life test to evaluate the reliability performance of the WLEDs. We performed the RA test at a high temperature and high relative humidity $(\mathrm{RH})$ of $85^{\circ} \mathrm{C}$ and $85 \mathrm{RH}$, respectively, with $120 \mathrm{~mA}$ on WLEDs containing $c$-SCPhC samples for $2500 \mathrm{~h}$, comparing the results with those from commercial WLEDs. A summary of the RA test, shown in Figure 5, indicates that the WLEDs containing $c$ $\mathrm{SCPhC}$ samples were nearly the same as commercial WLEDs; the $c$-SCPhCs deposited on the WLEDs were undamaged. The WLEDs show a weak drift of lumen maintenance due to the LuAG phosphor of the oxidation of $\mathrm{Ce}^{3+}$ to $\mathrm{Ce}^{4+}$ and the CASN phosphor of the oxidation of $\mathrm{Eu}^{2+}$ to $\mathrm{Eu}^{3+}$, respectively $[19,20]$. This failure analyses have been demonstrated by Lextar Electronics Corp. Therefore, this novel technique can 
TABLE 1: Optical characteristics of all light sources.

\begin{tabular}{lccccc}
\hline & & At $0.37 \mathrm{~W}$ & & \\
Light source & Luminous flux $(\mathrm{lm})$ & Luminous efficacy $(\mathrm{lm} / \mathrm{W})$ & CRI $(\mathrm{Ra})$ & CCT $(\mathrm{K})$ & CIE coordinate $(x, y)$ \\
\hline WLEDs & 53.2 & 144 & 82.3 & 3974 & $(0.3806,0.3744)$ \\
WLEDs with $c$-SCPhCs & 46.7 & 127 & 80.1 & 2960 & $(0.4428,0.4114)$ \\
$w$-WLEDs & 44.3 & 120 & 82.5 & 2842 & $(0.4464,0.4038)$ \\
\hline
\end{tabular}

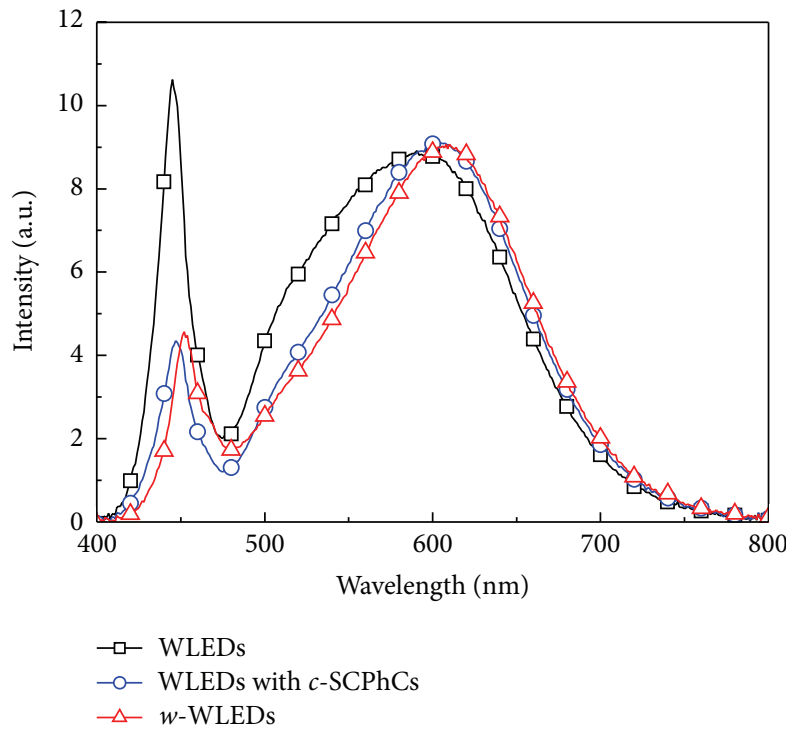

FIGURE 3: Luminescence spectra of the three types of WLEDs.

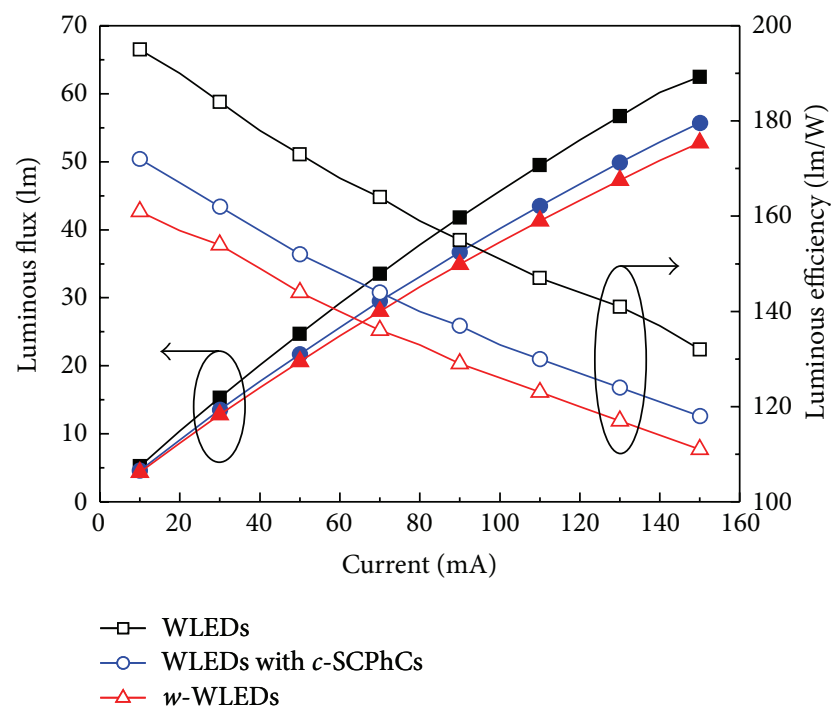

FIgURE 4: Current-dependent luminous flux ( $\operatorname{lm}$ ) and luminous efficiencies $(\mathrm{lm} / \mathrm{W})$ of the three types of WLEDs.

be used to enhance the WLEDs by reducing phosphor use and enhancing the luminous efficacy, thereby producing low-cost $w$-WLEDs.

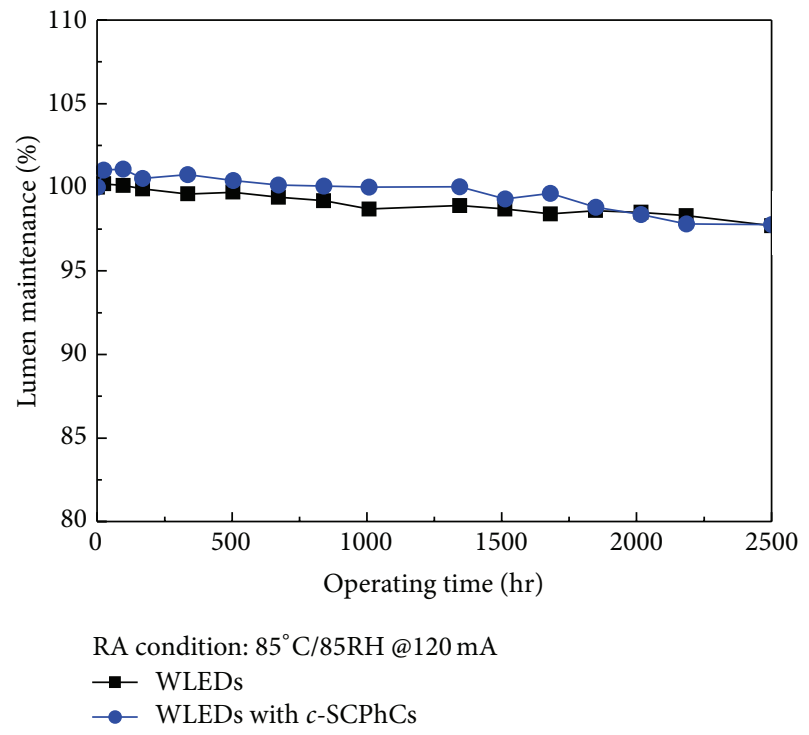

FIGURE 5: Degradation of lumen maintenance for the two types of WLEDs after the $85^{\circ} \mathrm{C} / 85 \mathrm{RH}$ with $120 \mathrm{~mA}$.

\section{Conclusion}

In this study, we developed a technique requiring little phosphor to fabricate low-cost $w$-WLEDs. The WLEDs containing $c$-SCPhCs in $120 \mathrm{~mA}$ produced a CCT of $2960 \mathrm{~K}$ and exhibited a CRI of 80 and a luminous flux of $46.7 \mathrm{~lm}$ (5.6\% more enhanced than that of the commercial $w$ WLEDs) without an increase in the concentration of the LuAG and CASN phosphors. In the WLEDs containing $c$ $\mathrm{SCPhCs}$, the $c$-SCPhCs thin films substantially modified the light emission spectrum because of the GNDF influence and multiple phosphor reemissions. In addition, the RA test showed no visible degradation in optical performance for WLEDs containing $c$-SCPhCs. This novel technique applied to WLEDs to produce $w$-WLEDs reduces fabrication costs and provides new options for designing safer light fixtures.

\section{Conflict of Interests}

The authors declare that there is no conflict of interests regarding the publication of this paper.

\section{Acknowledgments}

The authors gratefully acknowledge the financial support for this research by the National Science Council (NSC) and 
Ministry of Science and Technology (MOST) in Taiwan, under Grant nos. NSC102-2221-E-035-046, NSC102-2622E-035-030-CC2, MOST103-2221-E-035-029, and MOST1032622-E-035-007-CC2. The authors appreciate the Precision Instrument Support Center of Feng Chia University in providing the fabrication and measurement facilities.

\section{References}

[1] R.-H. Horng, P. Han, and D.-S. Wuu, "Phosphor-free white light from InGan blue and green light-emitting diode chips covered with semiconductor-conversion AlGaInP epilayer," IEEE Photonics Technology Letters, vol. 20, no. 13, pp. 1139-1141, 2008.

[2] X. Zou, K. M. Wong, X. Zhu, W. C. Chong, J. Ma, and K. M. $\mathrm{Lau}$, "High-performance green and yellow LEDs grown on $\mathrm{SiO}_{2}$ nanorod patterned GaN/Si templates," IEEE Electron Device Letters, vol. 34, no. 7, pp. 903-905, 2013.

[3] X.-H. Li, P. Zhu, G. Liu et al., "Light extraction efficiency enhancement of III-nitride light-emitting diodes by using 2-D close-packed $\mathrm{TiO}_{2}$ microsphere arrays," IEEE/OSA Journal of Display Technology, vol. 9, no. 5, pp. 324-332, 2013.

[4] K. Yamae, H. Tsuji, V. Kittichungchit, N. Ide, and T. Komoda, "Highly efficient white organic light-emitting diodes with over $100 \mathrm{~lm} / \mathrm{W}$ for next-generation solid-state lighting," Journal of the Society for Information Display, vol. 21, no. 12, pp. 529-540, 2013.

[5] H.-C. Kuo, C.-W. Hung, H.-C. Chen et al., "Patterned structure of remote phosphor for phosphor-converted white LEDs," Optics Express, vol. 19, no. 14, pp. A930-A936, 2011.

[6] J. H. Oh, S. J. Yang, Y.-G. Sung, and Y. R. Do, "Excellent color rendering indexes of multi-package white LEDs," Optics Express, vol. 20, no. 18, pp. 20276-20285, 2012.

[7] C.-F. Lai, C.-C. Chang, M.-J. Wang, and M.-K. Wu, "CCTand CRI-tuning of white light-emitting diodes using threedimensional non-close-packed colloidal photonic crystals with photonic stop-bands," Optics Express, vol. 21, no. 13, pp. A687A694, 2013.

[8] C. F. Lai, C. L. Hsieh, and C. J. Wu, "Light-spectrum modification of warm white-light-emitting diodes with 3D colloidal photonic crystals to approximate candlelight," Optics Letters, vol. 38, no. 18, pp. 3612-3615, 2013.

[9] C.-F. Lai, Y.-C. Lee, and C.-T. Kuo, "Saving phosphor by $150 \%$ and producing high color-rendering index candlelight LEDs containing composite photonic crystals," Journal of Lightwave Technology, vol. 32, no. 10, Article ID 6756966, pp. 1930-1935, 2014.

[10] P. Jiang, J. F. Bertone, K. S. Hwang, and V. L. Colvin, "Singlecrystal colloidal multilayers of controlled thickness," Chemistry of Materials, vol. 11, no. 8, pp. 2132-2140, 1999.

[11] G. G. Kumar, S. Senthilarasu, D. N. Lee et al., "Synthesis and characterization of aligned $\mathrm{SiO}_{2}$ nanosphere arrays: Spray method," Synthetic Metals, vol. 158, no. 17-18, pp. 684-687, 2008.

[12] T. M. Arantes, A. H. Pinto, E. R. Leite, E. Longo, and E. R. Camargo, "Synthesis and optimization of colloidal silica nanoparticles and their functionalization with methacrylic acid," Colloids and Surfaces A: Physicochemical and Engineering Aspects, vol. 415, pp. 209-217, 2012.

[13] E. Tian, L. Cui, J. Wang, Y. Song, and L. Jiang, "Tough photonic crystals fabricated by photo-crosslinkage of latex spheres," Macromolecular Rapid Communications, vol. 30, no. 7, pp. 509514, 2009.
[14] H. S. Lee, T. S. Shim, H. Hwang, S. M. Yang, and S. H. Kim, "Colloidal photonic crystals toward structural color palettes for security materials," Chemistry of Materials, vol. 25, no. 13, pp. 2684-2690, 2013.

[15] H. Li and Z. Xu, "Photoluminescence manipulation based on composite photonic crystal surfaces," Journal of Luminescence, vol. 143, pp. 288-292, 2013.

[16] C. López, "Materials aspects of photonic crystals," Advanced Materials, vol. 15, no. 20, pp. 1679-1704, 2003.

[17] C. I. Aguirre, E. Reguera, and A. Stein, "Tunable colors in opals and inverse opal photonic crystals," Advanced Functional Materials, vol. 20, no. 16, pp. 2565-2578, 2010.

[18] C. Zhaohui, Z. Qin, W. Kai, L. Xiaobing, and L. Sheng, "Reliability test and failure analysis of high power LED packages," Journal of Semiconductors, vol. 32, no. 1, Article ID 014007, 2011.

[19] R. Kasuya, T. Isobe, H. Kuma, and J. Katano, "Photoluminescence enhancement of PEG-modified YAG: $\mathrm{Ce}^{3+}$ nanocrystal phosphor prepared by glycothermal method," The Journal of Physical Chemistry B, vol. 109, no. 47, pp. 22126-22130, 2005.

[20] S. Mukherjee, V. Sudarsan, R. K. Vatsa, and A. K. Tyagi, "Luminescence studies on lanthanide ions $\left(\mathrm{Eu}^{3+}, \mathrm{Dy}^{3+}\right.$ and $\mathrm{Tb}^{3+}$ ) doped YAG:Ce nano-phosphors," Journal of Luminescence, vol. 129, no. 1, pp. 69-72, 2009. 

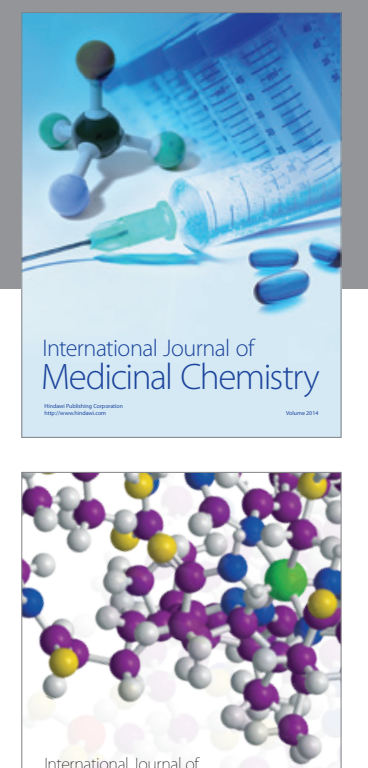

\section{Carbohydrate} Chemistry

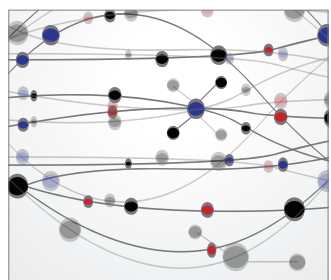

The Scientific World Journal
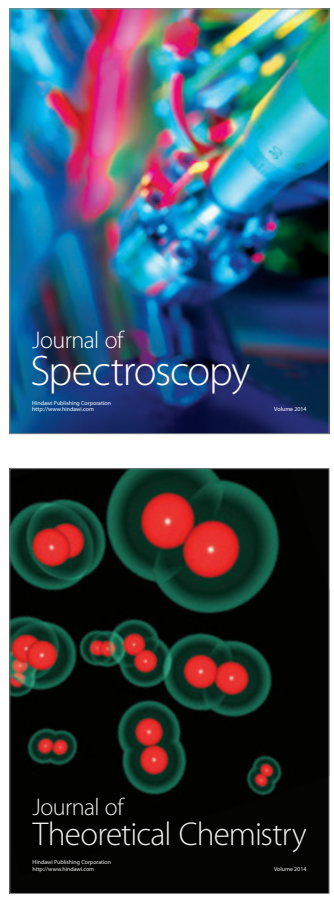
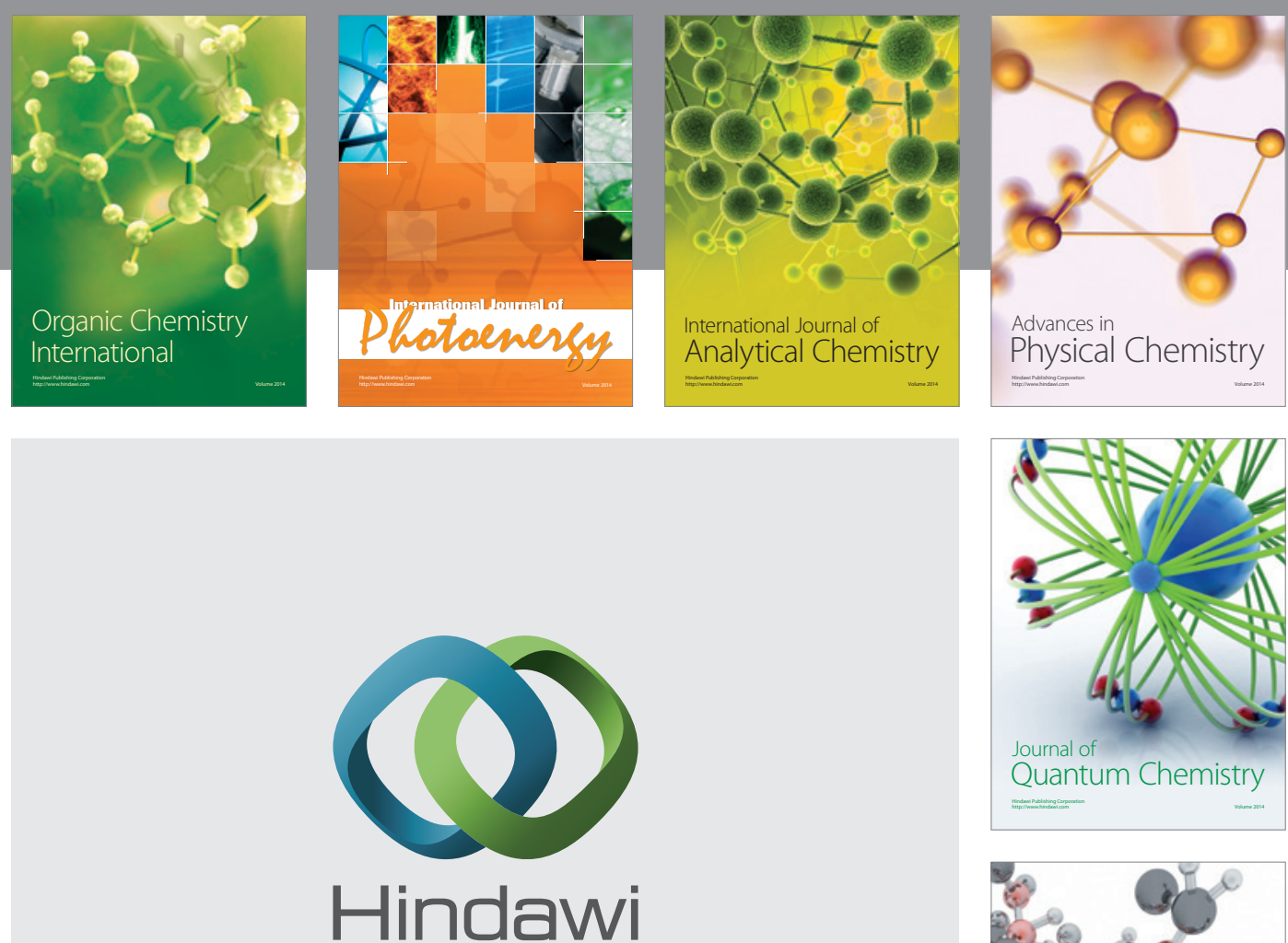

Submit your manuscripts at

http://www.hindawi.com

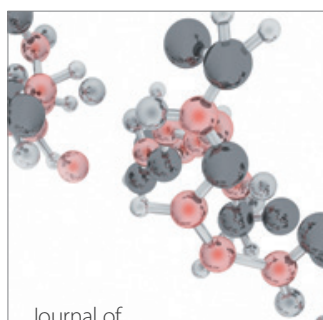

Analytical Methods

in Chemistry

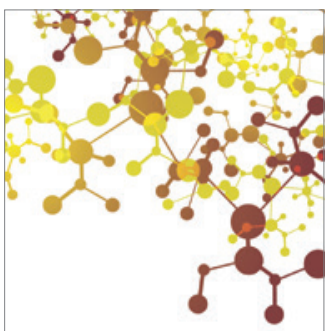

Journal of

Applied Chemistry

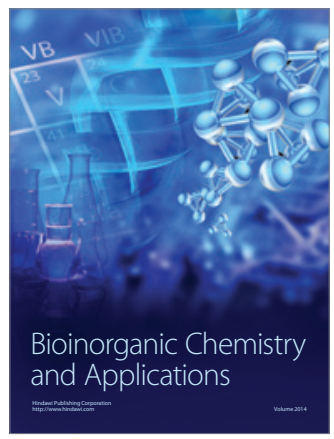

Inorganic Chemistry
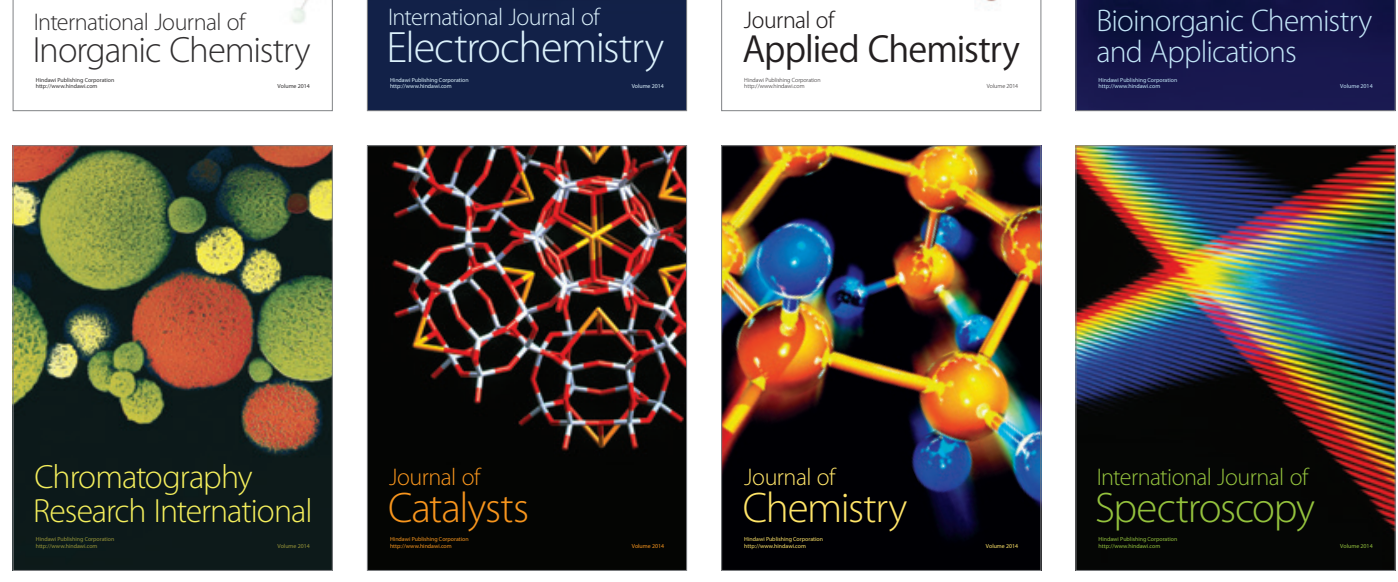